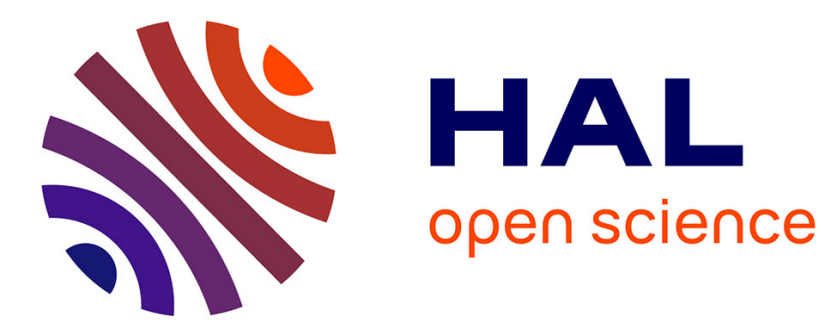

\title{
Sur de nouveaux conducteurs ioniques du lithium de structure boracite
}

Jean-Maurice Réau, Alain Levasseur, Guy Magniez, Bernard Calès, Paul

Hagenmuller

\section{- To cite this version:}

Jean-Maurice Réau, Alain Levasseur, Guy Magniez, Bernard Calès, Paul Hagenmuller. Sur de nouveaux conducteurs ioniques du lithium de structure boracite. Materials Research Bulletin, 1976, 11 (9), pp.1087-1090. 10.1016/0025-5408(76)90005-2 . hal-03480729

\section{HAL Id: hal-03480729 \\ https://hal.science/hal-03480729}

Submitted on 14 Dec 2021

HAL is a multi-disciplinary open access archive for the deposit and dissemination of scientific research documents, whether they are published or not. The documents may come from teaching and research institutions in France or abroad, or from public or private research centers.
L'archive ouverte pluridisciplinaire HAL, est destinée au dépôt et à la diffusion de documents scientifiques de niveau recherche, publiés ou non, émanant des établissements d'enseignement et de recherche français ou étrangers, des laboratoires publics ou privés. 
SUR DE NOUVEAUX CONDUCTEURS IONIQUES DU LITHIUM

DE STRUCTURE BORACITE

Jean-Maurice Réau, Alain Levasseur, Guy Magniez, Bernard Cales

Claude Fouassier et Paul Hagenmuller

Laboratolre de Chimie du Solide du C.N.R.S.

Universite de Bordeaux I

351, cours de la Liberation, 33405 Talence, France.

ABSTRACT

The electrical study of lithium boracites, which are electronic insulators, shows a high mobility of the $\mathrm{Li}^{+}$ cation. They are the first high isotropically conducting Li compounds.

Les etudes antérieures relatives aux electrolytes solides ont montré le grand intérêt présenté par des matériaux possédant une conductivité isotrope anionlque tels les zircones stabilisées $(1,2)$ et les fluorures dérives du type fluorine $(3,4,5)$ ou cationique tels 1 'iodure d'argent et ses derivés $(6,7)$ et les conducteurs tridimensionnels du sodium (8). Des considerations énergétiques et massiques ont orienté nos recherches vers le lithium plus lệer et susceptible de donner des tensions plus élevées que le sodium. Nous avons donc entrepris l'étude des boracites de lithium $\mathrm{Li}_{4} \mathrm{~B}_{7} \mathrm{O}_{12} \mathrm{X}(\mathrm{X}=\mathrm{Cl}, \mathrm{Br})$ isolants électroniques qu1 possèdent une structure tridimensionnelle et où les sites de lithium ne sont que partiellement occupés.

Nous avons représenté à la figure 1 l/8 de la mallie boracite dont la structure a eté determinée antérleurement au laboratoire (9). Les atomes de bore et d'oxygene forment un squelette tridimensionnel dans ies tunnels duquel se placent des octaedres $\mathrm{X}(\mathrm{LI})_{6}{ }_{6}(\mathrm{X}=\mathrm{Cl}, \mathrm{Br})$ lies par les sommets dans les trois directions de 1 espace. A la différence des trois atomes LiI qui ont une coordinence octaédrique, le quatrième atome de lithium LiII occupe un site tetraedrique formé par trols atomes d'oxygene et l'atome de chlore central : le taux d'occupation de celui-ci est de 258 . Cette structure présente done un grand interêt sur le plan de la moblilte lonique du lithium.

Les mesures electriques des boracltes de lithium ont eté effectues en courant alternatif entre $20 \mathrm{~Hz}$ et $200 \mathrm{kHz}$ par la methode des Impédances complexes. Celle-c1 permet en effet de séparer dans l'interprétation des mesures le rôle de l'électrolyte solide proprement dit de celui des electrodes et de déterminer ainsi avec précision la résistance ohmique des matérlaux considérés. 


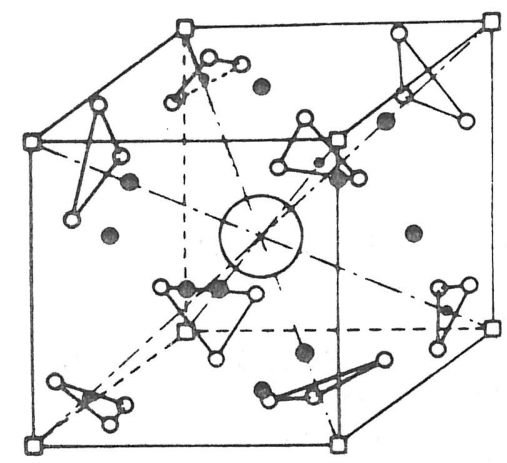

(8)

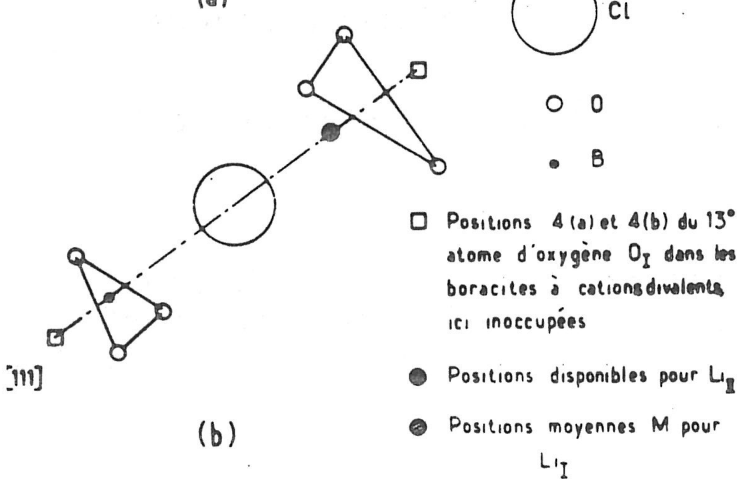

FIG. 1

Schéma structural de la boracite ( $1 / 8$ de mallie)

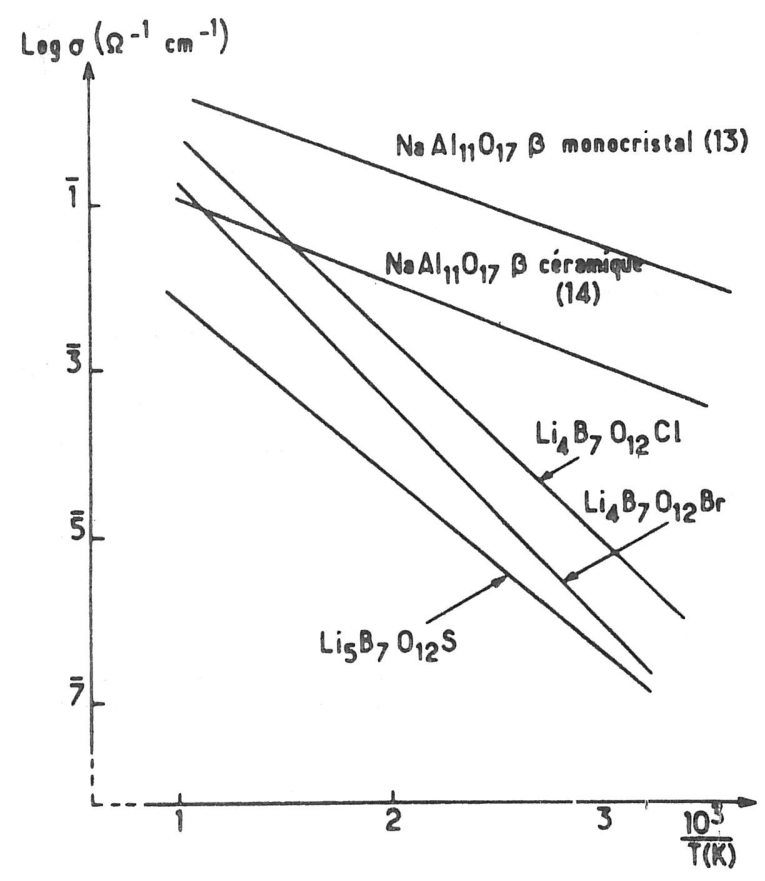

FIG. 2

Variation du logarithme de la conductivité avec la temperature des boracites de ilthium et de l'alumine $\beta$

Les échantillons utilisés sont des pastilles frittées à 840 et $800^{\circ} \mathrm{C}$ respectivement pour $\mathrm{I}_{4} \mathrm{~B}_{7} \mathrm{O}_{12} \mathrm{Cl}$ et $\mathrm{Li}_{4} \mathrm{~B}_{7} \mathrm{O}_{12} \mathrm{Br}$. Leur compacité est de l'ordre de 90 \& pour le matériau chloré et de $80 \%$ pour le produit brome. Les mesures électriques ont été effectuées entre 20 et $700^{\circ} \mathrm{C}$.

La figure 2 donne la variation du logarithme de la conductivite $\sigma$ en fonction de l'inverse de la temperature absolue pour les boracites $\mathrm{II}_{4} \mathrm{~B}_{7} \mathrm{O}_{12} \mathrm{Cl}$ et $\mathrm{II}_{4} \mathrm{~B}_{7} \mathrm{O}_{12} \mathrm{Br}$. Dans le domaine de temperrature envisage $\sigma$ est de la forme $\sigma=n q \mu_{0} \exp (-\Delta E / k T)$ ou $\mathrm{n}$ représente le nombre de porteurs de charge $q$ et de mobilite $\mu=\mu_{0} \exp (\Delta E / \mathrm{kT})$. $\Delta E$ est l'énergie d'activation liée au mécanisme de conduction.

Nous avons rassemble au tableau I les valeurs de la conductivite a $300^{\circ} \mathrm{C}$ et de l'énergie d'activation des materiaux étudiés. on peut attribuer la mobilite plus faible des lons $\mathrm{LI}^{+}$dans $\mathrm{LI}_{4} \mathrm{~B} 7 \mathrm{O}_{12} \mathrm{Br}$ a un effet sterique. Le remplacement dans $\mathrm{I}_{4} \mathrm{~B}_{7} \mathrm{O}_{12} \mathrm{Cl}$ de $\mathrm{I}^{\prime}$ ion $\mathrm{Cl}^{-}$ par l'Ion $\mathrm{Br}^{-}$conduit en effet à un leger affaiblissement de la Ila1son $L I$ - X qui devralt favoriser la mobllite des lons L1t, mais la présence die l'lon $\mathrm{Br}^{-}$de plus grande tallle $\left(r_{\mathrm{Br}^{-}}=1,96 \AA\right.$, $r_{\mathrm{Cl}^{-}}=1,81 \AA$ ) (10) entrave la diffusion des 10ns $\mathrm{L}^{+}$, I'augmentation de volume de la mallie elementaire ne compensant que partiellement cette gêne stêr1que (11). Cet effet est si important que la boracite lodée $\mathrm{Li}_{4} \mathrm{~B}_{7} \mathrm{O}_{12} \mathrm{I}$ ne peut etre préparée que sous pression de $30 \mathrm{~kb}$ (12). 
TABLEAU I

Conductivite et énergies d'activation des boracites de lithium

\begin{tabular}{lccc}
\hline & Compacite & $\begin{array}{c}\sigma \mathrm{a} 300^{\circ} \mathrm{C} \\
\left(\Omega^{-1} \mathrm{~cm}^{-1}\right)\end{array}$ & $\begin{array}{c}\Delta \mathrm{E} \\
(\mathrm{eV})\end{array}$ \\
\hline $\mathrm{Li}_{4} \mathrm{~B}_{7} \mathrm{O}_{12} \mathrm{Cl}$ & 908 & $10^{-2}$ & 0,49 \\
$\mathrm{Li}_{4} \mathrm{~B}_{7} \mathrm{O}_{12} \mathrm{Br}$ & 808 & $5.10^{-3}$ & 0,52 \\
$\mathrm{LI}_{5} \mathrm{~B}_{7} \mathrm{O}_{12} \mathrm{~S}$ & 708 & $3.10^{-4}$ & 0,42 \\
\hline
\end{tabular}

Pour ameliorer la moblitte des lons $\mathrm{Li}^{+}$dans la boracite,

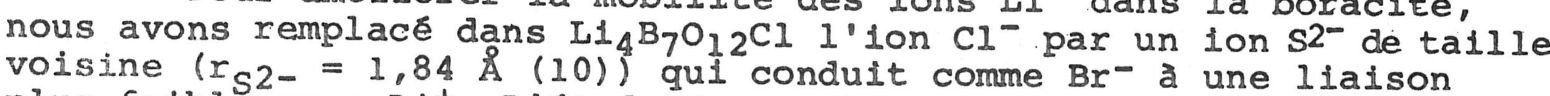
plus falble avec $\mathrm{Li}^{+}$. L'étude du système $\mathrm{B}_{2} \mathrm{O}_{3}-\mathrm{Li}_{2} \mathrm{O}-\mathrm{Li}_{2} \mathrm{~S}$ à $700^{\circ} \mathrm{C}$ a permis d'isoler une nouvelle boracite de lithium de formu-

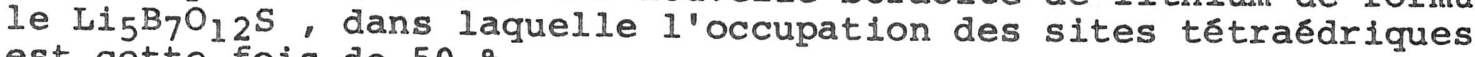
est cette fois de 50 \%

Nous avons porté à la flgure 2 la variation de la conductivite avec la température pour une pastille frittée de $\mathrm{Li}_{5} \mathrm{~B}_{7} \mathrm{O}_{12} \mathrm{~S}$. A même temperature la conductivite inferieure a celle des boracites chlorée et bromée résulte d'une plus falble compacité (tableau I) : celle-c1 $n$ 'excede pas en effet $70 \%$. En revanche $\mathrm{L}_{1} \mathrm{~B}_{7} \mathrm{O}_{12} \mathrm{~S}$ est caractérise par une energle d'activation plus faible $(\Delta E=0,42 \in \mathrm{E})$. Les ions $\mathrm{Li}^{+}$possedent donc dans ce matériau une plus grande mobi-
lite.

Nous avons reporte egalement à la flgure 2 la variation de la conductivite avec la temperature d'un monocristal et d'une cerramigue d'alumine $\beta$ de compacite volsine de $85 \%$. Les boracites de lithyum les plus performantes ont une conductivite aussi élevee que celle de l'alumine $B$ dans son domaine d'utilisation. Mais ces matériaux présentent par rapport à l'alumine $\beta$ l'avantage de comporter des temperatures de frittage beaucoup plus basses (15).

\section{Références}

1. C. Deportes, G. Robert et M. Forestier, Electrochimica Acta, 16,1003 (1971).

2. A. Hammou, C. Déportes et G. Robert, J. Chem. Phys., 7-8, 1162 (1971).

3. J.M. Réau, J. Claverie, G. Campet, C. Déportes, D. Ravaine, J.L. Souquet et A. Hammou, C.R. Acad. SC, 280 C, 325 (1975).

4. J.M. Réau, C. Lucat, G. Campet, J. Portier et A. Hammou, J. Solld State Chem., 17, 123 (1976).

5. C. Lucat, G. Campet, J. Claverie, J. Portier, J.M. Réau et P. Hagenmullex, Mat. Res. Bull., 11, 167 (1976).

6. A. Kv1st et A. Josefson, Z. Naturforsch., 23A, 625 (1968).

7. H. Wledesich et S. Gellar, The Chemistry of Extended Defects in Non-metallic Solids, Ed. L. Eyring and M. O'Keeffe, NorthHolland, Amsterdam, p. 629 (1970).

8. J.B. Goodenough, H.Y.P. Hong et J.A. Kafalas, Mat. Res. Bull., $11,203(1976)$. 
9. A. Levasseur, D.J. Lloyd, C. Fouassier et P. Hagenmuller, J. Solid State Chem., 8, 318 (1973).

10. R.D. Shannon et C.T. Prewitt, Acta Cryst., B25, 955 (1969).

11. A. Levasseur, C. Fouassier et P. Hagenmuller, Mat. Res. Bull., 6. 15 (1971). 12. W. Jeitschko et T.A. Bither, Z. Naturforschg, 27 (b) , II, 1423
(1972).

13. M.S. Whittingham et R.A. Huggins, J. Chem. Phys., 54, 414 (1971).

14. R.H. Radzilowski, Y.F. Yao et J.T. Kummer, J. Appl. Phys., 40, 4716 (1969).

15. J.M. Réau, A. Levasseur, G. Magniez, B. Calès et P. Hagenmuller, Brevet D.R.M.E. n 7611110 (1976).

Nous remercions la D.R.M.E. pour l'aide matérielle qu'elle a apportée à ce travail. 